\title{
Rational design of a CD4 mimic that inhibits HIV-1 entry and exposes cryptic neutralization epitopes
}

\author{
Loïc Martin ${ }^{1}$, François Stricher ${ }^{1}$, Dorothée Missé ${ }^{2}$, Francesca Sironi ${ }^{3}$, Martine Pugnière ${ }^{4}$, Philippe Barthe ${ }^{5}$, \\ Rafael Prado-Gotor ${ }^{5}$, Isabelle Freulon ${ }^{1}$, Xavier Magne ${ }^{2}$, Christian Roumestand ${ }^{5}$, André Ménez ${ }^{1}$, Paolo Lusso ${ }^{3}$, \\ Francisco Veas ${ }^{2}$, and Claudio Vita ${ }^{1 *}$
}

Published online 16 December 2002; doi:10.1038/nbt768

\begin{abstract}
The conserved surfaces of the human immunodeficiency virus (HIV)-1 envelope involved in receptor binding represent potential targets for the development of entry inhibitors and neutralizing antibodies. Using structural information on a CD4-gp120-17b antibody complex, we have designed a 27-amino acid CD4 mimic, CD4M33, that presents optimal interactions with gp120 and binds to viral particles and diverse HIV-1 envelopes with CD4-like affinity. This mini-CD4 inhibits infection of both immortalized and primary cells by HIV-1, including primary patient isolates that are generally resistant to inhibition by soluble CD4. Furthermore, CD4M33 possesses functional properties of CD4, including the ability to unmask conserved neutralization epitopes of gp120 that are cryptic on the unbound glycoprotein. CD4M33 is a prototype of inhibitors of HIV-1 entry and, in complex with envelope proteins, a potential component of vaccine formulations, or a molecular target in phage display technology to develop broad-spectrum neutralizing antibodies.
\end{abstract}

HIV-1 avoids the host immune surveillance by exposing hypervariable and heavily glycosylated regions on its exterior envelope ${ }^{1-3}$ while the conserved surfaces used to bind its cellular receptors are either located in recessed cavities (e.g., the CD4 binding site ${ }^{1,2}$ ) or exposed only after CD4 binding (e.g., the co-receptor ${ }^{4}$ binding site) as a result of conformational changes of the external envelope glycoprotein, gp120 (refs. 5, 6). Despite the mechanisms used by HIV-1 to protect its conserved binding surfaces, new clues to the design of effective gp120 inhibitors and neutralizing antibodies have come from recent crystallographic studies on gp120 in complex with CD4 and a Fab fragment of the neutralizing antibody $17 \mathrm{~b}$ (refs. 1,2).

In this complex, the CD4 surface binding to gp120 is centered on the CDR2-like loop, a protruding $\beta$-hairpin with its $C^{\prime \prime} \beta$-strand forming an antiparallel $\beta$-sheet with the gp120 $\beta 15$ strand. The CD4 binding site within gp120 is a large $\left(\sim 800 \AA^{2}\right)$ depression formed by conserved residues and presenting a deep hydrophobic pocket, denoted the 'Phe 43 cavity'1,2. Stabilizing interactions at the CD4 interface involve the Phe43 side chain, which occupies the entrance of the gp 120 cavity, and the Arg59 side chain, which forms a double H-bond with the gp120 Asp368 side chain ${ }^{1,2}$. The binding site for chemokine receptors, exposed only after CD4 binding ${ }^{4-6}$, is mostly formed by conserved residues of gp120 (refs. 3, 7) and overlaps the CD4-induced (CD4i) epitopes that are recognized by a class of monoclonal antibodies (mAbs), such as $17 \mathrm{~b}$ and $48 \mathrm{~d}$ (refs. 8,9 ), which block co-receptor binding ${ }^{5,6}$ and consequently infection, though with low potency 8 . . Recently, a human monoclonal Fab, X5, which targets the CD4i epitopes, has been selected by phage display technology and bio-panning against a CD4-gp120CCR5 complex ${ }^{10}$. This molecule potently neutralizes a broad spectrum of HIV-1 primary isolates from different subtypes ${ }^{10}$, suggesting that the CD4i epitopes are important targets of neutralizing antibodies ${ }^{10,11}$. Accordingly, HIV-1 envelope-sCD4 complexes, which represent intermediate structures present during the virus attachment to the cellular CD4 receptor and expose conserved cryptic epitopes, have been considered potential immunogens able to generate neutralizing antibodies ${ }^{12,13}$. This hypothesis has been confirmed in a recent study that showed that cross-linked HIV-1 envelope (gp120 or gp140)-sCD4 complexes, injected in rhesus macaques, elicited antibodies neutralizing a broad variety of primary viruses, regardless of co-receptor usage and genetic subtype ${ }^{14}$. The specificity of these neutralizing antibodies was shown to be primarily directed against envelope epitopes, although a substantial number of them were directed against the CD4 receptor. This represents an undesired property in candidate vaccines, because generating antibodies against the $\mathrm{CD} 4$ receptor may elicit an autoimmune response in humans.

Whereas the structural information on gp41 led to the design of peptide inhibitors and small molecules targeting the gp41 pre-fusion intermediates ${ }^{15,16}$, the available structural information on gp120 has not so far yielded effective small inhibitory molecules, although large immunoglobulin-related molecules targeting the CD4 binding site in gp120, such as the monoclonal IgGb12 antibody ${ }^{17}$, as well as both tetrameric ${ }^{18}$ and dodecameric ${ }^{19}$ forms of sCD4, have demonstrated promising results as HIV-1 inhibitors ${ }^{19,20}$.

${ }^{1}$ Department of Protein Engineering and Research, CEA Saclay, 91191 Gif-sur-Yvette, France. ${ }^{2}$ Retroviral and Molecular Immunology Laboratory, IRD/CNRS, 34094 Montpellier, France. ${ }^{3}$ Unit of Human Virology, San Raffaele Scientific Institute, 20132 Milan, Italy. ${ }^{4}$ Biotechnology and Pharmacology Institute, Faculty of Pharmacy, 34093 Montpellier, France. ${ }^{5}$ Structural Biochemistry Center, Faculty of Pharmacy, 34093 Montpellier, France. ${ }^{*}$ Corresponding author (claudio.vita@cea.fr). 
A

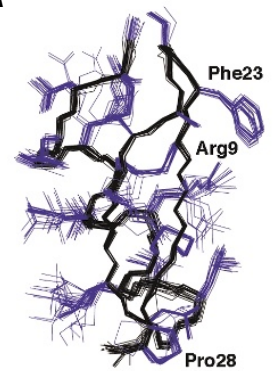

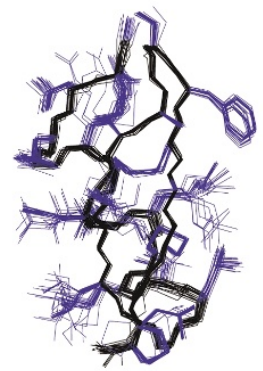

C

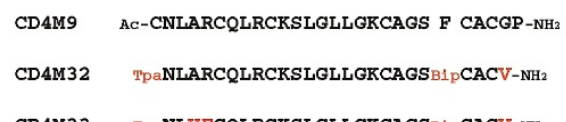

B

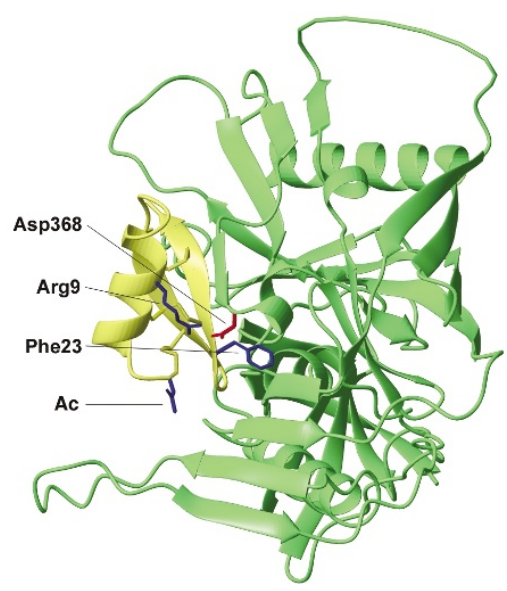

Figure 1. Structure, sequence, and computed complex of CD4 miniprotein. (A) Stereo view of the 30 low-energy NMR structures of CD4M9 miniprotein. Backbone and side-chain traces are in black and blue, respectively. (B) Theoretical model of CD4M9 miniprotein (yellow) in complex with gp120 (green), calculated according to the gp120 ${ }_{\mathrm{HXB} 2}-\mathrm{CD} 4$ complex crystallographic structure (PDB code, $1 \mathrm{~g} 9 \mathrm{~m})$. Amino acid side chains critical in the interactions are in blue stick for CD4M9 and red stick for gp120. Ac is the miniprotein $\mathrm{N}$-terminal acetyl group. The ribbon representation ${ }^{39}$ emphasizes the small size of the miniprotein that completely fills the CD4 binding site of gp120. (C) Sequence alignment of engineered miniprotein CD4M9 (ref. 23), CD4M32, and CD4M33, in one-letter amino acid code. Mutations introduced are in red. Tpa, Thiopropionic acid; Bip, biphenylalanine.

We had earlier designed a CD4 mimic (31 amino acids) presenting low, but specific gp120 affinity, by the transfer of the CDR2-like loop into the structurally equivalent loop of a small disulfide-stabilized structural scaffold, the scorpion toxin charybdotoxin ${ }^{21}$. A derivative of this mimic, added in large excess to gp120, was reported to induce exposure of CD4i epitopes ${ }^{22}$. By using the scyllatoxin scaffold, we then engineered a second miniprotein ${ }^{23}$, CD4M9 (28 amino acids), that was shown to inhibit gp 120 binding to soluble CD4 with a $50 \%$ inhibitory concentration $\left(\mathrm{IC}_{50}\right)$ that was still $\sim 100$-fold higher than that of native CD4, suggesting a suboptimal interaction with gp120. Taking advantage of the available structural information on the gp120-CD4 complex ${ }^{1,2}$, as well as the conformational stability and sequence permissiveness of the scaffold protein ${ }^{24,25}$, we redesigned the miniprotein binding surface so as to optimize gp120 binding interactions, producing a potent mini-CD4 with bona fide CD4-like properties. This optimized mini-CD4 inhibits infection of primary cells by primary clinical HIV-1 isolates and exposes cryptic neutralization epitopes of the viral envelope. Hence, this mini-CD4 has potential for therapeutic application in the inhibition of HIV-1 entry and, in complex with envelope proteins, for the development of neutralizing antibodies in vaccine formulations or for use as a molecular target in phage display technology to develop broad-spectrum neutralizing antibodies.

\section{Results}

Protein design. The engineering of a potent mini-CD4 was structure-guided, in that we first solved the three-dimensional structure of the CD4M9 miniprotein ${ }^{23}$ by NMR. The final 30 NMR low-energy structures (Fig. 1A) showed that the transferred functional site was well defined and the 19-28 $\beta$-hairpin of the CD4M9 average structure closely mimicked the corresponding CD4 39-48 region, which is central in gp120 binding, with only $0.7 \AA$ root-mean-square (RMS) deviation between the two backbones. On the basis of this structural similarity, we used the CD4M9 structure to model its complex with gp120, which we computed using the crystallographic structure of the gp120-CD4 complex ${ }^{2}$ as a template. In this model (Fig. 1B), the CD4 interactions that are critical for gp120 binding ${ }^{1,2}$ are also conserved. In particular, Phe 23 plugs the entrance of the gp120 'Phe43 cavity', the miniprotein $\beta$-strand $23-28$ forms an antiparallel $\beta$-sheet with gp120 $\beta 15$, and the Arg9 side chain forms a double $\mathrm{H}$-bond with the gp 120 Asp368 carboxyl. After complex formation, the miniprotein $\mathrm{N}$ terminus (including its $\mathrm{N}$-acetyl group) moves by $>1 \AA$. Thus, when binding to gp120 in a CD4-like conformation, CD4M9 undergoes unfavorable steric contacts at its $\mathrm{N}$ terminus.

Based on these findings, the N-terminal residue was replaced by thiopropionic acid (Tpa), which poses only minimal steric hindrance. Furthermore, to increase interaction with the apolar 'Phe43 cavity', Phe23 was replaced by a biphenylalanine (Bip) residue, which presents an additional hydrophobic phenyl moiety. Finally, to stabilize the C-terminal $\beta$-strand and to favor the formation of a $\beta$-sheet interaction with the $\beta 15$ strand of gp120, the C-terminal Gly27-Pro28 sequence was replaced by a valine residue. Each single mutation produced an increase in gp120 binding affinity (Table 1), as revealed by competition enzyme-linked immunosorbent assay (ELISA). The simultaneous incorporation of the three mutations (Tpa1, Bip23, Val27) into a CD4M9 triple mutant (designated CD4M32, Fig. 1C) resulted in $\sim 40$ - to 80 fold enhancement in the gp120 binding affinity (depending on the isolate) as compared with CD4M9 (Table 1). To protect the disulfide bond 1-19 from the solvent and to stabilize the helical region, two additional mutations, $\mathrm{A} 4 \mathrm{H}$ and $\mathrm{R} 5 \mathrm{~F}$, were then introduced into a new miniprotein, designated CD4M33 (Fig. 1C). Despite maintaining $67 \%$ of the original scyllatoxin sequence, the derivative bearing all five mutations, CD4M33, did not show any substantial binding affinity to the potassium channel (not shown), which is the cellular target of scyllatoxin. In competition ELISA, this molecule inhibited sCD4 binding to both gp $120_{\mathrm{HXB} 2}$, derived from a laboratory-adapted CXCR4-using (X4) strain, and gp120 JRFL, from a CCR5-using (R5) strain, with $7.5 \mathrm{nM}$ and $4.0 \mathrm{nM} \mathrm{IC}_{50}$, respectively (Table 1 ). These values are comparable to those obtained with sCD4 (Table 1) and indicate that the engineered CD4M33 mimic binds to gp120 with a CD4 native-like affinity.

gp120 Binding. Direct binding of CD4M33 to various HIV-1 recombinant envelope gp120 glycoproteins was demonstrated using an optical biosensor system, based on surface plasmon resonance ${ }^{22,26}$. Real-time monitoring of gp120 ${ }_{\mathrm{HXB} 2}(\mathrm{X} 4)$ or gp120 $0_{\mathrm{W} 61 \mathrm{D}}(\mathrm{R} 5)$ binding to CD4M33 (Fig. 2A, B) yielded the following association/dissociation

\begin{tabular}{lcc}
\hline \multicolumn{3}{c}{ Table 1. Binding data of CD4 miniproteins and sCD4 } \\
\hline Competitor & IC $_{50}$ (gp120 $\left._{\mathrm{HXB} \text { ) }}\right)^{\mathrm{a}}$ & IC $_{50}$ (gp120JRFL \\
\hline CD4M9 & $1.6 \mu \mathrm{M}$ & $2.4 \mu \mathrm{M}$ \\
[Tpa1]CD4M9 & $0.22 \mu \mathrm{M}$ & $0.3 \mu \mathrm{M}$ \\
[Bip23]CD4M9 & $0.90 \mu \mathrm{M}$ & $1.5 \mu \mathrm{M}$ \\
[Val27]CD4M9 & $0.96 \mu \mathrm{M}$ & $1.6 \mu \mathrm{M}$ \\
CD4M32 & $37.5 \mathrm{nM}$ & $30.0 \mathrm{nM}$ \\
CD4M33 & $7.5 \mathrm{nM}$ & $4.0 \mathrm{nM}$ \\
sCD4 & $2.0 \mathrm{nM}$ & $1.1 \mathrm{nM}$ \\
CD4M33-F23A & $>>100 \mu \mathrm{M}$ & $>>100 \mu \mathrm{M}$ \\
\hline
\end{tabular}

alC $_{50}$, Half-maximal (50\%) inhibitory concentration obtained in competition ELISA. Standard deviations were within $15 \%$ of the mean.

${ }^{b}$ Recombinant two-domain CD4. 
Research Article

rate and equilibrium dissociation constants: $k_{\mathrm{a}}=4.54 \pm 0.04 \times 10^{4}$ $\mathrm{M}^{-1} \mathrm{~s}^{-1}, k_{\mathrm{d}}=3.70 \pm 0.02 \times 10^{-4} \mathrm{~s}^{-1}$, and $K_{\mathrm{d}}=8.15 \pm 0.08 \mathrm{nM}$ for $\operatorname{gp} 120_{\mathrm{HXB} 2}\left(\chi^{2}\right.$ of 1.88$) ; k_{\mathrm{a}}=5.44 \pm 0.06 \times 10^{4} \mathrm{M}^{-1} \mathrm{~s}^{-1}, k_{\mathrm{d}}=6.70 \pm 0.03 \times$ $10^{-4} \mathrm{~s}^{-1}$, and $K_{\mathrm{d}}=12.3 \pm 0.1 \mathrm{nM}$ for gp120 $0_{\mathrm{W61D}}\left(\chi^{2}\right.$ of 0.76$)$. CD4M33 was able to bind gp120 from a variety of isolates, such as SF2(X4), IIIB(X4), JRFL(R5), and BaL(R5), with $K_{\mathrm{d}}$ values within the 1-20 nM range (data not shown), which are comparable to those determined for native sCD4 (refs. 26, 27).

Virus inhibition. Next, we analyzed the ability of the CD4M33 mimic to inhibit HIV-1 cell-cell fusion and infection of cells expressing CD4 and either the CCR5 or CXCR4 co-receptor. CD4M33 effectively blocked fusion of HeLa cells expressing the HXB2 (X4), JRFL (R5), or 89.6 (X4/R5) envelopes with HeLa or NIH 3T3 target cells expressing CD4 and the appropriate co-receptors, CXCR4 or CCR5, with an $\mathrm{IC}_{50}$ of $0.8 \pm 0.09 \mu \mathrm{M}(2.3 \mu \mathrm{g} / \mathrm{ml}), 7.0 \pm 0.6 \mu \mathrm{M}(20.5 \mu \mathrm{g} / \mathrm{ml})$, and $7.6 \pm 0.3 \mu \mathrm{M}(22.3 \mu \mathrm{g} / \mathrm{ml})$, respectively, similarly to sCD4, which inhibited fusion with an $\mathrm{IC}_{50}$ of $0.05 \pm 0.004 \mu \mathrm{M}(1.0 \mu \mathrm{g} / \mathrm{ml}), 4.0 \pm$ $1.0 \mu \mathrm{M}(80 \mu \mathrm{g} / \mathrm{ml})$, and $1.1 \pm 0.2 \mu \mathrm{M}(22 \mu \mathrm{g} / \mathrm{ml})$, respectively. CD4M33 effectively inhibited infection by different HIV-1 strains, including HIV-1 $1_{\text {НХв2 }}$ (X4), HIV-1 $1_{\text {IIIB }}$ (X4), HIV-1 $1_{\text {YU2 }}$ (R5), and HIV$1_{\mathrm{BaL}}(\mathrm{R} 5)$ both in continuous cell lines, such as PM1 and co-receptortransfected $\mathrm{HeLa}$, and in primary peripheral blood mononuclear cells (PBMC), with $\mathrm{IC}_{50}$ in the nanomolar range (Table 2). Importantly, CD4M33 also inhibited infection by primary HIV-1 isolates (PI), including a pure CCR5-using isolate (224-18), two dual-tropic isolates (204-2, 193-21) that use CCR5 and CXCR4, and a promiscuous isolate (196-1) that uses CCR5, CCR3, and CXCR4 for entry ${ }^{28}$ (Table 2). As a specificity control, we tested the variant CD4M33-F23A, in which the critical residue Bip23 was substituted by an alanine residue. This sole substitution was sufficient to abolish completely both the binding to gp120 (Table 1) and the antiviral activity (not shown). Therefore, the CD4M33 antiviral activity seems to be closely related to its high gp120 binding affinity. Interestingly, in both PM1 and PBMC assays, the CD4M33 concentrations required for $50 \%$ virus inhibition were substantially lower than those required for $\mathrm{sCD} 4$ and were independent of the isolate

\begin{tabular}{llll}
\hline \multicolumn{4}{l}{ Table 2. Antiviral activity of CD4M33 and sCD4 } \\
\hline HIV-1 isolates & $\begin{array}{l}\text { Co-receptor } \\
\text { (clade B) }\end{array}$ & CD4M33 & sCD4 \\
& & $\mathrm{IC}_{50} \pm \mathrm{s.d}$. & $\mathrm{IC}_{50} \pm \mathrm{s.d}$. \\
& $\mathrm{nM}(\mathrm{ng} / \mathrm{ml})$ & $\mathrm{nM}(\mathrm{ng} / \mathrm{ml})$ \\
\hline
\end{tabular}

\section{HeLa}

$\mathrm{HXB2}$

YU2

PI 224-18

\section{X4}

$\mathrm{R} 5$

R5

PI 204-2 X4/R5

PI 196-1

$\begin{aligned} 1.6(4.6) & \pm 0.8 \\ 5.2(15.1) & \pm 0.8 \\ 6.5(18.9) & \pm 0.9 \\ 2.0(5.8) & \pm 0.3 \\ 11.0(31.9) & \pm 0.9\end{aligned}$

$11.0(31.9) \pm 0.9$

PM1

\begin{tabular}{llcc} 
IIIB & X4 & $1.9(5.5) \pm 1$ & $53(1,060) \pm 1$ \\
$\mathrm{BaL}$ & $\mathrm{R} 5$ & $6.7(19.6) \pm 3$ & $211(4,220) \pm 15$ \\
$\mathrm{PI}^{\mathrm{b}} 224-18$ & $\mathrm{R} 5$ & $69(202) \pm 21$ & $339(6,780) \pm 8$ \\
$\mathrm{PI}^{\mathrm{b}} 193-21$ & $\mathrm{X} 4 / \mathrm{R} 5$ & $6.7(19.6) \pm 2$ & $81(1,620) \pm 4$ \\
& & & \\
PBMC & & & \\
IIIB & $\mathrm{X} 4$ & $3.8(11.1) \pm 1.6$ & $95(1,890) \pm 22$ \\
$\mathrm{BaL}$ & $\mathrm{R} 5$ & $151(442) \pm 34$ & $500(9,965) \pm 140$ \\
$\mathrm{PI}^{b} 224-18$ & $\mathrm{R} 5$ & $1475(4,322) \pm 1100$ & $3,835(76,726) \pm 1,450$ \\
$\mathrm{PI}^{b} 193-21$ & $\mathrm{X} 4 / \mathrm{R} 5$ & $69(202) \pm 28$ & $515(10,335) \pm 130$ \\
\hline
\end{tabular}

asCD4, Two-domain recombinant human CD4. ${ }^{\mathrm{b}} \mathrm{PI}$, Primary isolate ${ }^{28}$. At the concentrations used, no cytotoxicity of CD4M33 has been observed. Inactive CD4M33-F23A does not show any virus inhibition activity (data not shown). In PM1, inhibitory concentrations were calculated at day 3 for $\mathrm{HIV}-1_{\text {IIIB }}$ and $\mathrm{HIV}-1_{\mathrm{BaL}}$, and at day 7 for PIs. In PBMC, inhibitory concentrations were calculated at day 3 for all isolates.
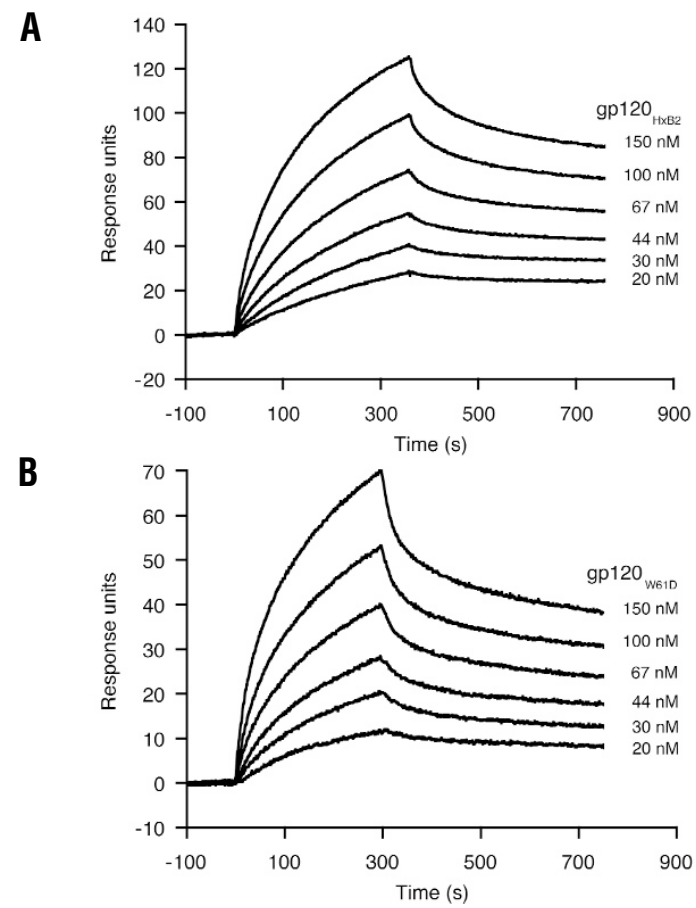

Figure 2. Binding activity of the CD4M33 miniprotein. Sensorgrams of recombinant (A) gp120 $0_{\mathrm{HXB}}$ and (B) gp120 ${ }_{\text {W61D }}$ binding to immobilized CD4M33, obtained by optical biosensor technology. Each gp120 solution was injected at the indicated concentrations.

tropism (Table 2). The R5 primary isolate 224-18 (ref. 28) was consistently the most resistant to neutralization, requiring $69 \mathrm{nM}(202$ $\mathrm{ng} / \mathrm{ml})$ in PM1 and $1.4 \mu \mathrm{M}(4.3 \mu \mathrm{g} / \mathrm{ml})$ in PBMC to attain $50 \%$ virus neutralization by CD4M33. Of note, in PBMC the antiviral potency of CD4M33 was about $1 \log$ lower compared with that measured in the PM1 cell line, whereas sCD4 inhibited at even higher concentrations. Both mini-CD4 and sCD4 inhibited cell-cell fusion in our hands at markedly higher concentrations compared with those required to inhibit virus-cell fusion. This result likely reflects the cell fusion assay used, based on vaccinia technology ${ }^{29}$, which is characterized by maximal surface expression of both envelope and receptor/co-receptor complexes mediating fusion, and thus requires higher inhibitor concentrations.

Conformational changes in HIV envelopes. We then investigated whether the CD4M33 mimic is capable of unmasking gp120 epitopes that are cryptic on the native glycoprotein, but can be induced by CD4 binding (CD4i epitopes). As detected by optical biosensor technology, gp $120_{\mathrm{HXB} 2}$ was found to bind efficiently to the $48 \mathrm{~d}$ antibody in the presence of either CD4M33 or sCD4 (Fig. 3A), with calculated $K_{\mathrm{d}}$ values of $3.2 \pm 0.5 \mathrm{nM}$ and $1.3 \pm 0.1 \mathrm{nM}$, respectively. In contrast, in the presence of the less potent CD4M9 miniprotein, the inactive CD4M33-F23A variant, or alone, this gp120 showed very little, if any, binding to $48 \mathrm{~d}$ (Fig. 3A). Similarly, CD4M33 was found to increase the binding affinity of the X4 envelope glycoprotein SF2, or the R5 glycoproteins W61D and JRFL to 48d, as well as to immobilized $17 \mathrm{~b}$ mAb (not shown). Notably, effective binding of HIV- $1_{\mathrm{HXB} 2}$ viral particles that had been inactivated by Aldrithiol-2 (AT-2), which modifies only free thiol groups but preserves full structural integrity of envelope proteins ${ }^{30}$, to $48 \mathrm{~d}$ (or to $17 \mathrm{~b}$, not shown) was also detected in the presence of CD4M33, as demonstrated by RTPCR conducted on viral particles recovered from the dissociation phase of optical biosensor experiments (Fig. 3B). This finding suggests that CD4M33 binds not only to monomeric recombinant gp120 proteins but also to native HIV-1 envelope on viral particles 
A

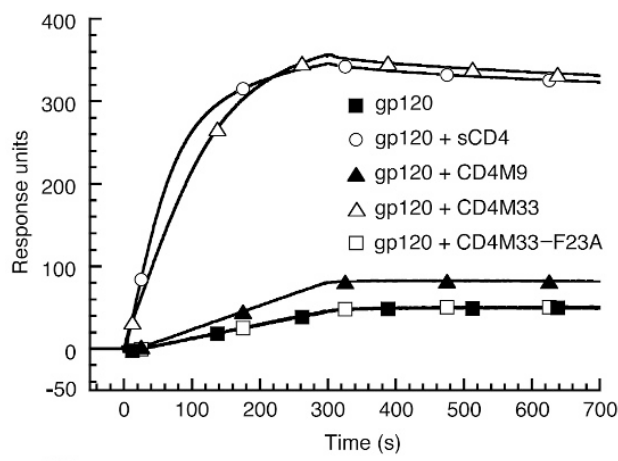

B

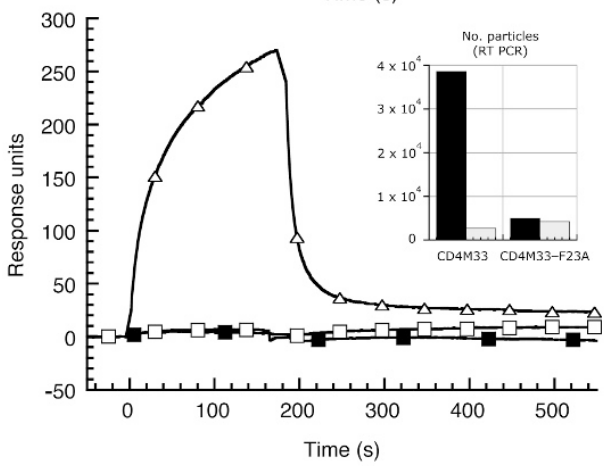

C

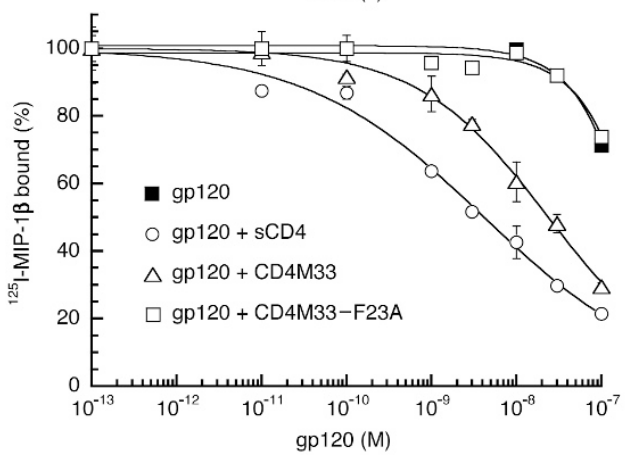

Figure 3. Conformational changes in gp120 and HIV-1 envelope induced by CD4M33 binding. (A) Sensorgrams of gp120 ${ }_{\mathrm{HXB} 2}(125 \mathrm{nM})$ binding to immobilized $48 \mathrm{~d} \mathrm{mAb}$ obtained by optical biosensor technology in the absence $(\square)$ or in the presence of threefold molar excess SCD4 $(O)$, CD4M33 $(\triangle)$, inactive CD4M33-F23A ( $\square$ ), or CD4M9 (A). (B) Binding sensorgrams of $\mathrm{HIV}-1_{\mathrm{HXB} 2}$ particles in the absence $(\boldsymbol{\square})$ or in the presence of CD4M33 $(\triangle)$ or CD4M33-F23A $(\square)$ to immobilized $48 \mathrm{~d}$ mAb obtained by optical biosensor technology. (Inset) RT-PCR analysis of material recovered after binding of HIV-1 particles to both immobilized $48 \mathrm{~d} \mathrm{mAb}$ (filled bars) and irrelevant $2 \mathrm{C} 2 \mathrm{mAb}$ (open bars), in the presence of CD4M33 (left) or inactive CD4M33-F23A (right). (C) Inhibition of ${ }^{125}$ I-MIP$1 \beta$ binding to CCR5-expressing cells (CHO-K1) by gp120 ${ }_{\text {W61D }}$ alone $(\mathbb{\square})$ or in the presence of $100 \mathrm{nM} \mathrm{sCD} 4(O), \operatorname{CD} 4 \mathrm{M} 33(\triangle)$, and inactive CD4M33-F23A ( $\square$ ). Data were normalized for nonspecific (0\%) and specific binding in the absence of competitor (100\%).

and, furthermore, it induces gp120 conformational changes that expose CD4i viral epitopes.

Next, we investigated whether these conformational changes also increase the affinity of gp120 for chemokine receptors ${ }^{5,6}$. To this end, the competition between the R5 HIV-1 envelope W61D and the chemokine MIP- $1 \beta$ for CCR5 binding was studied in the presence of either CD4M33 or sCD4. Binding of radiolabeled MIP-1 $\beta$ was inhibited by gp $120_{\mathrm{W} 61 \mathrm{D}}$ in the presence of CD4M33 $\left(\mathrm{IC}_{50}=2.3 \pm 0.1 \times 10^{-8} \mathrm{M}\right)$ or sCD4 $\left(\mathrm{IC}_{50}=4.1 \pm 0.6 \times 10^{-9} \mathrm{M}\right)$. Low binding was observed with gp120 $0_{\text {W61D }}$ alone or in the presence of the inactive CD4M33-F23A mutant (Fig. 3C). Thus, the ability of CD4M33 to expose CD4i epi- topes is associated with a capacity to expose the envelope co-receptor binding site, though slightly less (sixfold) efficiently than with sCD4. This finding was also confirmed using a modified cell fusion assay ${ }^{29}$, in which our mini-CD4, similarly to sCD4 was able to induce fusion of HIV-1 envelope expressing cells with target cells expressing only the co-receptor (CCR5 or CXCR4) but lacking membrane-bound fulllength CD4 (data not shown). Altogether, these data show that the interaction of CD4M33 with gp120 is functionally similar to that of native sCD4.

\section{Discussion}

The optimized mini-CD4 herein described represents a prototype of a gp120-targeted antiviral agent that exhibits functional characteristics of native human CD4, while offering important advantages due to its small size $(2.9 \mathrm{kDa})$ and constrained structure. Like native CD4, CD4M33 binds with high affinity to the CD4 binding site on the HIV-1 exterior envelope. Moreover, it blocks the entry and infection of primary cells by HIV-1 clinical isolates in vitro. Like sCD4, it induces conformational changes in a variety of gp120 envelope recombinant proteins, as well as in native (oligomeric) envelopes on HIV-1, exposing conserved viral epitopes that are targets of neutralizing antibodies ${ }^{8-11}$. However, in contrast to sCD4 $(40 \mathrm{kDa})$ and other larger oligomeric CD4-based constructs ${ }^{18,19}$, CD4M33 is small and possesses a constrained and disulfidestabilized structure. These properties may favor an easier access to the viral envelope CD4 binding sites that, especially in primary isolates, may be partially occluded by hypervariable loops ${ }^{1,2,31}$. This may explain why its antiviral activity is superior to that of sCD4. In addition, similar to the precursor, CD4M9 (ref. 25), CD4M33 is stable in strong denaturant conditions (acidic $\mathrm{pH}, 90{ }^{\circ} \mathrm{C}, 4 \mathrm{M}$ guanidine hydrochloride) and more resistant than sCD4 to degradation by proteases (e.g., trypsin, chymotrypsin, and cathepsin $\mathrm{H}$, data not shown).

Although sCD4 has been tested in clinical trials against HIV, the results have been disappointing ${ }^{32}$ as a consequence of its poor performance against primary HIV isolates. By contrast, in vitro data with the more stable CD4IgG2 construct ${ }^{18,20}$ or the dodecameric CD4-Ig fusion protein ${ }^{19}$ are promising, and the former is being tested in clinical trials ${ }^{33}$. Thus, although the HIV-inhibitory potency of CD4M33 has to be confirmed with a larger panel of clinical isolates from different subtypes, its in vitro activity profile against eight different HIV-1 isolates suggests that this mini-CD4 or appropriate derivative molecules might have a therapeutic potential as an antiviral agent, especially as a topical microbicide or in the prevention of mother-to-child virus transmission. Nonetheless, although peptides as drugs present critical limitations, such as poor bioavailability and potential immune response, the proof-ofconcept that viral entry can be successfully blocked in vivo by a synthetic peptide, T-20, has already been established ${ }^{34}$. Moreover, the CD4M33 antiviral activity also validates the CD4 binding site in gp120 as a potential target for small organic molecules, structurally related or unrelated to the mini-CD4 (ref. 25). In this respect, this mini-CD4 can be fluorescently labeled and used in screening tests to identify inhibitory molecules (C. Vita et al., manuscript in preparation). Given the increased emergence of HIV strains resistant to the current multidrug therapy ${ }^{35,36}$, new antiviral agents working with different mechanisms and on different viral targets will be highly desirable.

Finally, as suggested by the recent study on rhesus macaques, immunization with crosslinked envelope-CD4 complexes ${ }^{14}$, our optimized mini-CD4, which is a fully functional CD4 substitute and exposes cryptic conserved viral epitopes more efficiently than its precursor CD4M9 miniprotein ${ }^{23}$, might find use, when complexed and crosslinked to different envelope forms, in producing stable 
entry intermediates that can be used as immunogens in animal immunization to elicit broadly cross-reactive neutralizing antibodies. A distinctive advantage of this CD4 mimic is that its limited epitope sharing with human CD4 should prevent an anti-CD4 immune response, which was detected when envelope-CD4 complexes were used $^{14}$, thus avoiding potential autoimmune responses-a feature that is of fundamental importance for vaccine development. Finally, these mini-CD4-envelope complexes, with the possible addition of a co-receptor (CCR5 or CXCR4) molecule ${ }^{10}$, could potentially be used in bio-panning experiments with a Fab phage library to develop broadly cross-reactive HIV-1 neutralizing antibodies ${ }^{10}$. An advantage of this mini-CD4 is that its small size will allow better access to sites close to the CD4 binding site; thus, this construct may result in in vitro selection of neutralizing antibodies with new specificities.

\section{Experimental protocol}

Peptide synthesis. The mini-CD4 peptides were synthesized by solid-phase methods as described ${ }^{24,25}$. Biotin was specifically incorporated at Lys11 in CD4M33, by using the 1-(4,4-dimethyl-2,6-dioxocyclohex-1-ylidene)ethyl (Dde) protecting group during peptide synthesis and subsequent coupling of biotinamido-caproate- $N$-hydroxysuccinimide ester, after Dde removal by $2 \%$ hydrazine ${ }^{25}$. Synthetic peptides were purified by reverse-phase HPLC and their identity verified by electrospray mass spectrometry.

Structure calculation. Structures were determined from standard two-dimensional ${ }^{1} \mathrm{H}$-NMR experiments (TOCSY, DQF-COSY, and NOESY) recorded at $600 \mathrm{MHz}$ (Bruker AMX-600) on a $4 \mathrm{mM}$ protein sample, $\mathrm{pH} 3.5$, either in $95 \%$ $\mathrm{H}_{2} \mathrm{O}-5 \% \mathrm{D}_{2} \mathrm{O}$ or in $100 \% \mathrm{D}_{2} \mathrm{O}$ solutions, at $25^{\circ} \mathrm{C}$. Standard methods were used for recording and analyzing spectra, deriving distance and angle restraints $\mathrm{s}^{37}$. A total of 999 three-dimensional structures were generated from 297 NOEs and 27 angular restraints using the torsion angle dynamics protocol of DYANA ${ }^{38}$. The 30 conformers with the lowest final target function value $\left(<0.11 \AA^{2}\right)$ were energy-minimized with the AMBER program. Average global RMS deviations calculated for backbone and heavy atoms are $0.41 \pm 0.17 \AA$ and $1.12 \pm 0.31 \AA$, respectively, after refinement.

Molecular modeling. The crystallographic structure of gp120-CD4 complex ${ }^{2}$ (PDB code $1 \mathrm{~g} 9 \mathrm{~m}$ ) was used as a template to dock the miniprotein CD4M9 on gp120. The CD4 was then replaced by the lowest energy CD4M9 NMR structure by superimposing the 19-28 backbone residues of CD4M9 to those of 39-48 CD4 sequence. Energy minimization (side chains only) was followed by a 25 ps simulated annealing procedure in which the relative weights for the nonbonded and torsion energy terms gradually increase from 0.1 to 1.0. Finally, energy minimization was applied to CD4M9 and gp120 side chains. This procedure was repeated ten times, and the complex with the minimal energy retained. Molmol software ${ }^{39}$ was used for molecular graphics.

Binding assays. Inhibition of gp120-CD4 interaction was measured in competition ELISA, by using the D7324 antibody (Aalto Bio Reagents, Dublin, Ireland) to capture gp120, as described ${ }^{23,40}$. Competition CCR5 binding assays were done as described ${ }^{5,6,41}$ by using CHO-K1 cells stably expressing CCR5 (ref. 42).

Surface plasmon resonance biosensor analysis. Experiments were conducted at $25{ }^{\circ} \mathrm{C}$ with $20 \mu \mathrm{l} / \mathrm{min}$ flow rate in HBS (HEPES-buffered saline, $3 \mathrm{mM}$ EDTA, $0.05 \%$ Biacore surfactant, $\mathrm{pH}$ 7.4) with a BIACORE 2000 instrument (Biacore AB, Uppsala, Sweden). For direct binding of gp120 glycoproteins to CD4 miniprotein, a B1 sensor chip, precoated with 700 response units (RU) of streptavidin, was used to immobilize biotinylated-CD4M33 (50 RU). For binding of gp120 to $48 \mathrm{~d}$ and $17 \mathrm{~b}$ mAbs, they were immobilized at $1,500 \mathrm{RU}$ by the amine coupling kit, provided by the manufacturer. The gp $120_{\mathrm{HXB} 2}$ $(125 \mathrm{nM})$ and sCD4 or CD4 miniproteins $(375 \mathrm{nM})$ were premixed at $37^{\circ} \mathrm{C}$ for $60 \mathrm{~min}$ before injection at $20 \mu \mathrm{l} / \mathrm{min}$. All the sensorgrams were corrected by subtracting the signal from reference flow cell and, in the case of sCD4, normalized to the miniprotein mass. Flow-independent kinetic parameters showed the absence of mass transport and rebinding effects. The association and dissociation phase data were fitted to a Langmuir 1:1 global fitting model. For HIV-1 particles binding experiments, the supernatant of acutely infected cells was clarified by centrifugation and filtration $(0.45 \mu \mathrm{m}$; Gelman Laboratory, Ann Arbor, MI); concentrated virus preparations were produced, and shed gp120 was washed out by centrifugation using a $10^{6}$-kDa-cutoff
Vivaspin 20 concentrator (Sartorius, Göttingen, Germany). Viral particles were then inactivated with $500 \mu \mathrm{M}$ AT-2 (ref. 30) (Sigma, St. Louis, MO) at 4 ${ }^{\circ} \mathrm{C}$ overnight. Residual AT-2 was eliminated by ultracentrifugation, and viruses were resuspended in PBS (20 mM phosphate buffer, $\mathrm{NaCl} 150 \mathrm{mM}, \mathrm{pH}$ 7.4). Virus suspension was incubated for $30 \mathrm{~min}$ at $37^{\circ} \mathrm{C}$ with either $\mathrm{CD} 4 \mathrm{M} 33$ or CD4M33-F23A, before injection on immobilized $48 \mathrm{~d} \mathrm{mAb}$.

Cell fusion assays. Effector cells (HeLa) infected with vaccinia vectors expressing biologically different HIV-1 envelopes (the X4 HXB2, the R5 JRFL, or the R5X4 89.6) (ref. 30) were mixed for $2 \mathrm{~h}$ with target cells infected with vaccinia vectors expressing the appropriate co-receptor (NIH3T3-CCR5 or HeLa-CXCR4) and CD4. Effector cells were always co-infected with a vaccinia vector expressing bacteriophage T7 RNA polymerase, whereas target cells were always co-infected with a vaccinia vector expressing the $l a c Z$ reporter gene under the control of the T7 promoter. Cell fusion was determined by measurement of $\beta$-galactosidase activity in nonionic detergent cell lysates. The assay was done as described ${ }^{29}$.

HIV-1 infection assays. A single-cycle infection assay was used with HeLa P4 or HeLa-P4 CCR5 target cells $\mathrm{s}^{43}$ that constitutively express CD4, co-receptors, and $\beta$-galactosidase under the control of HIV-1-LTR. Virus at 500 times the $50 \%$ tissue culture infective dose $\left(\mathrm{TCID}_{50}\right)$ was mixed with $\mathrm{CD} 4$-based molecules for $1 \mathrm{~h}$ at $25^{\circ} \mathrm{C}$; then $2.5 \times 10^{4}$ target cells were added, incubated for an additional hour, and extensively washed. Subsequently, cells were incubated in culture DMEM, 10\% FCS (Gibco BRL, Cergy Pontoise, France) in $5 \% \mathrm{CO}_{2}$ atmosphere at $37^{\circ} \mathrm{C}$ for $24 \mathrm{~h}$. $\beta$-Galactosidase activity was then measured as described $^{44}$. Experiments were done in triplicate and repeated twice. The $\mathrm{IC}_{50}$ was calculated by GraphPad3.

For the acute infection assay in PM1 cell line ${ }^{45}$, an immortalized $\mathrm{CD}^{+}{ }^{+} \mathrm{CCR} 5^{+} \mathrm{CXCR} 4^{+} \mathrm{T}$-cell clone susceptible to a wide variety of HIV-1 strains, the cells were seeded at $2.5 \times 10^{4}$ cells/well into flat-bottom 96-well plates in $200 \mu \mathrm{l}$ of complete RPMI medium. Cells were then exposed to the viral stocks $\left(\sim 50 \mathrm{TCID}_{50} /\right.$ well $)$ pretreated or not with serial dilutions of inhibitors for $30 \mathrm{~min}$. After overnight incubation at $37^{\circ} \mathrm{C}$, the cells were washed twice and recultured in complete RPMI medium supplemented with the appropriate inhibitors. After 3, 5, and $7 \mathrm{~d}$, the culture supernatant was removed for p24 antigen testing and replaced with fresh medium containing the appropriate inhibitors. The extracellular p24 concentrations were measured by capture ELISA, using a sandwich of specific antibodies (Aalto Bio Reagents).

For the acute infection assay in primary cells, normal PBMC were isolated by Ficoll-Hypaque (Pharmacia, Uppsala, Sweden) gradient centrifugation from concentrated leukocytes of healthy donors and stimulated with phytohemagglutinin (PHA; Sigma) and interleukin-2 (IL-2) in RPMI 1640 medium (Gibco) supplemented with 10\% FCS (Biochrom, Berlin, Germany), $2 \mathrm{mM}$ glutamine, and antibiotics. PBMC were infected by exposing cells $\left(10^{5} /\right.$ well $)$ to the viral stocks ( $50 \mathrm{TCID}_{50} /$ well), in duplicate 96 -well roundbottom microtiter plates, in $200 \mu \mathrm{l}$ complete RPMI medium supplemented with $10 \%$ heat-inactivated FBS and $50 \mathrm{U} / \mathrm{ml}$ recombinant IL-2, in the presence or absence of inhibitors. After incubation at $37^{\circ} \mathrm{C}$ for $16 \mathrm{~h}$, the wells were washed twice and recultured in complete medium with or without inhibitors. After 48 h, 75\% of the supernatant was removed for HIV-1 p24 antigen measurement and replaced by an equal volume of fresh medium containing IL-2 and the respective inhibitors. Virus replication was assayed at days 3 and 5 after infection by p 24 antigen ELISA, using commercial antibodies (Aalto Bio Reagents).

HIV-1 particle recovery. A suspension of AT-2-inactivated viral particles ( $80 \mu \mathrm{l}, 10^{9}$ particles), prepared as above, were preincubated with either 27.7 $\mu \mathrm{M}$ of CD4M33 or inactive CD4M33-F23A at $37^{\circ} \mathrm{C}$ during 30 min and then injected on immobilized CD4i (48d, 17b) mAbs or on anti-tyrosine kinase, $2 \mathrm{C} 2$ irrelevant $\mathrm{mAb}$ at $25^{\circ} \mathrm{C}$. Virus retained by $\mathrm{CD} 4 \mathrm{i} \mathrm{mAbs}$ on the sensor chip was dissociated by a treatment with $50 \mu \mathrm{l}$ of $1.5 \mathrm{M}$ formic acid, collected, neutralized with $50 \mu \mathrm{l}$ of $1.5 \mathrm{M}$ Tris- $\mathrm{HCl}, \mathrm{pH} 8.8$, and HIV-1 quantitative RTPCR processed to assess the viral particle number (COBAS, Amplicor, HIV monitor; Roche Basel, Switzerland).

\section{Acknowledgments}

We thank N. Schulke (Progenics, Tarrytown, NY) for four-domain sCD4 and gp120 from R5-tropic JRFL; I. Srivastava (Chiron Co., Emeryville, CA) for recombinant gp120 from X4-tropic SF2; S. Leow (Pharmacia, Kalamazoo, MI) for two-domain sCD4; M. Parmentier (University of Brussels, Belgium) for 
CHO-K1 CCR5 cell line. We thank E.A. Berger (Laboratory of Viral Diseases (LVD), NIH, Bethesda, MD) for providing vaccinia virus constructs, $G$. Scarlatti (Unit of Viral Biology and Transmission, San Raffaele Scientific Institute, Milan, Italy) for providing primary HIV-1 isolates, and O. Combes, D. Seiller (Commissariat Energie Atomique (CEA), Saclay, France), M. Cerutti, M. Secondy (Institut Recherche Developpement (IRD), Montpellier, France), for their assistance and comments. We thank the late J.C. Mani for his assistance and discussion in optical biosensor experiments. We acknowledge the Centralised Facility for AIDS Reagents, NIBSC, UK (EU Programme

1. Kwong, P.D. et al. Structure of an HIV gp120 envelope glycoprotein in complex with the CD4 receptor and a neutralizing antibody. Nature 393, 648-659 (1998).

2. Kwong, P.D. et al. Structures of HIV-1 gp120 Envelope glycoproteins from laboratory adapted and primary isolates. Structure Fold. Des. 8, 1329-1339 (2000).

3. Wyatt, R. et al. The antigenic structure of the HIV gp120 envelope glycoprotein. Nature 393, 705-711 (1998).

4. Berger, E.A., Murphy, P.M. \& Farber, J.M. Chemokine receptors as HIV-1 coreceptors: roles in viral entry, tropism, and disease. Annu. Rev. Immunol. 17, 657-700 (1999).

5. Wu, L. et al. CD4-induced interaction of primary HIV-1 gp120 glycoproteins with the chemokine receptor CCR-5. Nature 14, 179-183 (1996).

6. Trkola, A. et al. CD4-dependent, antibody-sensitive interactions between HIV-1 and its co-receptor CCR-5. Nature 384, 184-187 (1996).

7. Rizzuto, C.D. et al. A conserved HIV gp120 glycoprotein structure involved in chemokine receptor binding. Science 280, 1949-1953 (1998).

8. Thali, M. et al. Characterization of conserved human immunodeficiency virus type 1 gp120 neutralization epitopes exposed upon gp120-CD4 binding. J. Virol. 67, 3978-3988 (1993)

9. Sullivan, N. et al. CD4-Induced conformational changes in the human immunodeficiency virus type 1 gp120 glycoprotein: consequences for virus entry and neutralization. J. Virol. 72, 4694-4703 (1998).

10. Moulard, M. et al. Broadly cross-reactive HIV-1-neutralizing human monoclonal Fab selected for binding to gp120-CD4-CCR5 complexes. Proc. Natl. Acad. Sci. USA 99, 6913-6918 (2002).

11. Wyatt, R. \& Sodroski, J. The HIV-1 envelope glycoproteins: fusogens, antigens, and immunogens. Science 280, 1884-1888 (1998).

12. Kang, C.Y, Hariharan, K., Nara, P.L., Sodroski, J. \& Moore, J.P. Immunization with a soluble CD4-gp120 complex preferentially induces neutralizing anti-human immunodeficiency virus type 1 antibodies directed to conformation-dependent epitopes of gp120. J. Virol. 68, 5854-5862 (1994).

13. DeVico, A., Silver, A., Thronton, A.M., Sarngadharan, M.G. \& Pal, R. Covalently crosslinked complexes of human immunodeficiency virus type 1 (HIV-1) gp120 and CD4 receptor elicit a neutralizing immune response that includes antibodies selective for primary virus isolates. Virology 218, 258-263 (1996).

14. Fouts, T. et al. Crosslinked HIV-1 envelope-CD4 receptor complexes elicit broadly cross-reactive neutralizing antibodies in rhesus macaques. Proc. Natl. Acad. Sci. USA 99, 11842-11847 (2002).

15. Chan, D.C. \& Kim, P.S. HIV entry and its inhibition. Cel/ 93, 681-684 (1998).

16. Eckert, D.M. \& Kim, P.S. Mechanisms of viral membrane fusion and its inhibition. Annu. Rev. Biochem. 70, 777-810 (2001).

17. Gauduin, M.C. et al. Effective ex vivo neutralization of human immunodeficiency virus type 1 in plasma by recombinant immunoglobulin molecules. J. Virol. 70, 2586-2592 (1996)

18. Allaway, G.P. et al. Expression and characterization of CD4-IgG2, a novel heterotetramer that neutralizes primary HIV type 1 isolates. AIDS Res. Hum. Retroviruses 11, 533-539 (1995).

19. Arthos, J. et al. Biochemical and biological characterization of a dodecameric CD4-Ig fusion protein. J. Biol. Chem. 277, 11456-11464 (2002).

20. Trkola, A. et al. Cross-clade neutralization of primary isolates of human immunodeficiency virus type 1 by human monoclonal antibodies and tetrameric CD4-lgG. J. Virol. 69, 6609-6617 (1995).

21. Drakopoulou, E., Vizzavona, J. \& Vita, C. Engineering a CD4 mimetic inhibiting the binding of the human immunodeficiency virus-1 (HIV-1) envelope glycoprotein gp120 to human lymphocyte CD4 by the transfer of a CD4 functional site to a small natural scaffold. Lett. Pep. Sci. 5, 241-245 (1998).

22. Zhang, W. et al. Conformational changes of gp120 in epitopes near the CCR5 binding site are induced by CD4 and a CD4 miniprotein mimetic. Biochemistry $\mathbf{3 8 ,}$ 9405-9416 (1999)

23. Vita, C. et al. Rational engineering of a miniprotein that reproduces the core of the
EVA/MRC, Grant Nr QLK2-CT-1999-00609 and GP828102) for recombinant gp120 from R5 W61D, anti-human CD4 L120.3 and 17b, 48d monoclonal antibody. We thank the French Agence Nationale de Recherches sur le SIDA (ANRS) and Ensemble Contre le SIDA-SIDACTION for financial support.

Competing interests statement

The authors declare that they have no competing financial interests.

Received 18 June 2002; accepted 4 November 2002
CD4 site interacting with HIV-1 envelope glycoprotein. Proc. Natl. Acad. Sci. USA 96, 13091-13096 (1999).

24. Vita, C., Roumestand, C., Toma, F. \& Ménez, A. Scorpion toxins as natural scaffolds for protein engineering. Proc. Natl. Acad. Sci. USA 92, 6404-6408 (1995).

25. Martin, L., Barthe, P., Combes, O., Roumestand, C. \& Vita, C. Engineering novel bioactive mini-proteins on natural scaffolds. Tetrahedron 56, 9451-9460 (2000).

26. Myszka, D.G. et al. Energetics of the HIV gp120-CD4 binding reaction. Proc. Natl. Acad. Sci. USA 97, 9026-9031 (2000).

27. Wu, H. et al. Kinetic and structural analysis of mutant CD4 receptors that are defective in HIV gp120 binding. Proc. Natl. Acad. Sci. USA 93, 15030-15035 (1996).

28. Scarlatti, G. et al. In vivo evolution of HIV-1 co-receptor usage and sensitivity to chemokine-mediated suppression. Nat. Med. 11, 1259-1265 (1997).

29. Salzwedel, K., Smith, E.D., Dey, B. \& Berger, E.A. Sequential CD4-coreceptor interactions in human immunodeficiency virus type 1 Env function: soluble CD4 activates Env for coreceptor-dependent fusion and reveals blocking activities of antibodies against cryptic conserved epitopes on gp120. J. Virol. 74, 326-333 (2000).

30. Rossio, J.L. et al. Inactivation of human immunodeficiency virus type 1 infectivity with preservation of conformational and functional integrity of virion surface proteins. J. Virol. 72, 7992-8001 (1998).

31. Kwong, P.D., Wyatt, R., Sattentau, Q.J., Sodroski, J. \& Hendrickson, W.A. Oligomeric modeling and electrostatic analysis of the gp120 envelope glycoprotein of human immunodeficiency virus. J. Virol. 74, 1961-1972 (2000).

32. Daar, E.S., Li, X.L., Moudgil, T. \& Ho, D.D. High concentrations of recombinant soluble CD4 are required to neutralize primary human immunodeficiency virus type 1 isolates. Proc Natl Acad Sci USA 87, 6574-6578 (1990).

33. Shearer, W.T. et al. Recombinant CD4-lgG2 in human immunodeficiency virus type 1-infected children: phase $1 / 2$ study. The Pediatric AIDS Clinical Trials Group Protocol 351 Study Team. J. Infect. Dis. 182, 1774-1779 (2000).

34. Kilby, J.M. et al. Potent suppression of HIV-1 replication in humans by T-20, a peptide inhibitor of gp41-mediated virus entry. Nat. Med. 4, 1302-1307 (1998).

35. Little, S.J. et al. Reduced antiretroviral drug susceptibility among patients with primary HIV infection. JAMA 282, 1142-1149 (1999).

36. Solomon, H. et al. Prevalence of HIV-1 resistant to antiretroviral drugs in 81 individuals newly infected by sexual contact or injecting drug use. Investigators of the Quebec Primary Infection Study. AIDS 14, F17-23 (2000).

37. Wüthrich, K. NMR of Proteins and Nucleic Acids (Wiley, New York, 1986).

38. Guntert, P., Mumenthaler, C. \& Wüthrich, K. Torsion angle dynamics for NMR structure calculation with the new program DYANA. J. Mol. Biol. 273, 283-298 (1997).

39. Koradi, R., Billeter, M. \& Wüthrich, K. MOLMOL: a program for display and analysis of macromolecular structures. J. Mol. Graphics 14, 51-55 (1996).

40. Moore, J.P. Simple methods for monitoring HIV-1 and HIV-2 gp120 binding to SCD4 by ELISA: HIV-2 has a 25 -fold lower affinity than HIV-1 for SCD4. AIDS 4 , 297-303 (1990).

41. Martin, L. et al. Structural and functional analysis of the RANTES-glycosaminoglycans interactions. Biochemistry 40, 6303-6318 (2001).

42. Samson, M., Labbe, O., Mollereau, C., Vassart, G. \& Parmentier, M. Molecular cloning and functional expression of a new human CC-chemokine receptor gene. Biochemistry 35, 3362-3367 (1996)

43. Charneau, P. et al. HIV-1 reverse transcription. A termination step at the center of the genome. J. Mol. Biol. 241, 651-662 (1994).

44. Misse, D. et al. Dissociation of the CD4 and CXCR4 binding properties of human immunodeficiency virus type 1 gp120 by deletion of the first putative $\alpha$-helical conserved structure. J. Virol. 72, 7280-7288 (1998)

45. Lusso, P. et al. Growth of macrophage-tropic and primary human immunodeficiency virus type 1 (HIV-1) isolates in a unique CD4 ${ }^{+}$T-cell clone (PM1): failure to downregulate CD4 and to interfere with cell-line-tropic HIV-1. J. Virol. 69, 3712-3720 (1995) 